\title{
Cuban or Cuban American
}

National Cancer Institute

\section{Source}

National Cancer Institute. Cuban or Cuban American. NCI Thesaurus. Code C67115.

Denotes a person from or of Cuba or an American citizen of Cuban descent. 